\title{
THE ANTHROPOGENIC REFUGE AREAS FOR BEE FLORA IN AGRICULTURAL LANDSCAPE
}

\author{
${ }^{1}$ Bożena Denisow, ${ }^{2}$ Malgorzata Wrzesień
}

\begin{abstract}
${ }^{1}$ Department of Botany, Agricultural University, Akademicka 15 str., 20950 Lublin, Poland e mail: bozena.denisow@ar.lublin.pl
${ }^{2}$ Department of Geobotany, Institute of Biology, Maria Curie Skłodowska University, Akademicka 19, 20033 Lublin, Poland; e mail: mseptember@tlen.pl
\end{abstract}

Received: 15.03 .2007

$$
\text { S u m m a r y }
$$

The investigations were carried out in 20022006 in two municipalities of Konopnica and Jastków close to the western part of Lublin, Poland. The aim of the study was to identify the con dition of bee flora and determine the floristic richness depending on the biotops in agricultural landscape. Flora of the anthropoge nic refuge areas consists of 214 species, among them $80 \%$ were flow taxons. Apophytes (162 species $78 \%$ ) predominated on all types of biotops under consideration. Perennials predominated in flora of boundary strips and bush communities. Mainly annual (40\%) and biennial (15\%) species comprise the flora of fallows. A great number of bee taxons represent meadow, segetal or rude ral communities. Most of flow plants compose loose patches, but their successive blooming ensures a source of food for Apoidea from early spring till the end of summer. The flora of boundary strips, bush communities and fallows significantly increases the biodiversity in agricultural landscape. The treatments including sowing, mainly on fallows with nectariferous and polleniferous species, would enrich generally weak flows in highly agricultural landscape.

Key words: refuge areas, agricultural landscape, pollenferous and nectariferous taxons, environs of Lublin

\section{INTRODUCTION}

The opinion about pollution of agricultural landscape is generally accepted (van Opstal, 2000). Rapid changes of agricultural technologies encouraged farmers to abandon traditional husbandry methods and mixed farming systems in favour of intensive enterprises with pesticides, herbicides, fungicides and other chemicals. New technologies influenced both segetal and ruderal flora. The advantages of synantropic taxons in agroecosystems are widely discussed. The benefits include soil enrichment with nutrients, positive alleopathic effects. A great number of weeds are known as bee plants and can be important from two main points of view, as honey raw material and the development of wild bees colonies (Parish and Bazzaz, 1979; Jabłoński and Kołtowski, 1995; Warakomska, 1997; Wr ó blew s ka, 2002). Unfortunately, the expansion of specialisation and intensification caused unprecedented changes of forage base (Corbet et al. 1991; Jabłońs ki, 2000).

An increasing emphasis is now being placed upon replacement of environmentally distracted policies with other ones which encourage natural resources (Dostatny, 2006). The one involving the emergence of contemporary agri-environmental strategies should save and rebuild distracted fragments of unarable lands. The aim of the present study was to identify the condition of bee flora and determine the floristic richness depending on the biotops in agricultural landscape.

\section{MATERIAL AND METHODS}

\section{The characteristic of the study area}

Field studies were carried out in the years 2002 2006 in two municipalities of Konopnica and Jastków close to the western part of the Lublin agglomeration (Fig. 1). The region is situated in the central-northern part of the Lublin Upland and is a part of the Nałęczów Plateau and the Bełżyce Plain. The area under investigations forms a broad belt between $51^{\circ} 96^{\prime}-50^{\circ} 86^{\prime} \mathrm{N}$ and of $20^{\circ} 28^{\prime}-22^{\circ} 6^{\prime} \mathrm{E}$ and extends on a highly undulated area at 180-252 m above see level. Most soils are claydusty and sandy-dusty or loess-originated brown and grey-brown (Turski et al. 1993).

The potential natural vegetation of the area is composed of the Ciemiega stream valley with a large area of Phragmitetea class. In the close neighbourhood, there are wet, rarely mown meadows with communities from the Molinion and Calthion alliances. Higher located meadows with standard mowing management 
are characteristic to Arrhenatherion elatioris. The most valuable as a source of bee flora is Cirsietum rivularis with the predominance of Cirsium rivulare $(70 \%)$ and Polygonum bistorta (30\%). Small surface areas are occupied by phytocenoses from Scheuchzerio-Caricetea fuscae. The fragments of Ribo nigri-Alnetum are present mainly in the eastern part of the Jastków municipality. Some other natural woodland complexes of varied size occur as isolated areas in different parts of the studied area. Usually, the forests represent fragmented forms of Querco-Fagetea and are reduced in species. Rapid economic changes after the transformation in 1989 caused strong anthropogenic pressure and are partly responsible for an increase of fallows to approx. $18 \%$ of arable lands. The cultivated area includes $7500 \mathrm{ha}$, the farming is still intensive and the mosaic structure of landscape characteristic for the eastern part of Poland is maintained (Figs. 2, 3). Most of the cultivated area is under cereals (63-68\%) and about $18-22 \%$ is occupied by root crops (potato fields and sugar beet). An abundant nectar and pollen flow was observed only in May, during the blooming of both orchards and meadows. Entomophilous crops such as rape plantations and perennial papilionaceous were very rare.

\section{Methods}

Observations of the entire flora were made up separately on field margins, bush communities and fallows, every two-three weeks during vegetation to take seasonal blooming aspects and to assign the time and length of blooming. The list of nectariferous and polleniferous taxons was established on the basis of data from literature (Warak o m s ka, 1995,1997; Wróblew s ka, 2002) and according to own observations. The record provides taxonomic nomenclature by Mirek et al. (2002) and the description of each taxon including its life span, geographic - historical status (Zając 1979; Zając et. al. 1998), sinecological group (Matus zk i ew i c z, 2001), and average time of blooming. The intensity of Apoidea foraging was estimated as averages on the basis of 30 min observations concerning each species, during sunny weather in the most intense foraging hours (10.00$-14.00 \mathrm{EET}$ ) in full blooming period. The following range for bee visits was applied: weak $-0-1 \cdot \mathrm{m}^{-2}$; medium $2-4 \cdot \mathrm{m}^{-2} ; \operatorname{good}>5 \cdot \mathrm{m}^{-2}$.

\section{RESULTS}

The data concerning the diversity and distribution of bee flora in different agricultural biotops are given in Tab. 1.

Presently, the flora of the anthropogenic refuge areas consists of 214 species belonging to 39 families of which the most abundant are Asteraceae, Fabaceae, Rosaceae, Caryophyllaceae, Brassicaceae and Lamiaceae (Fig. 5). The great majority of taxons occurred on fal- lows - 153 species, among them $73 \%$ were identified as good bee plants. Then, 124 different vascular plants were present on boundary strips, including 103 nectariferous and polleniferous. In the flora of margins, a high grass participation $(2 \%)$ was observed which is probably connected with a high level of fertilisation on adjacent fields. The least abundant and most floristically stable were bush communities with only 34 species recorded and recognised as flow species.

Apophytes (162 species - 78\%) predominated on all types of biotops under consideration (Fig. 5). Alien species occurred less frequently and were represented by archeophytes (36 species $-17 \%$ ), epecophytes (7 species $-3,3 \%$ ), and of short duration agriophytes (only 4 species $-2 \%$ ). The complete absence of efemerophytes indicates a very low coefficient of synantropisation in the analyzed biotops. Interestingly, a considerable increase of development in the last 15 years and the transformation of arable lands into fallows did not cause the inflow of alien species.

Perennials predominated among species recorded on different refuge areas in the studied agricultural landscape (Fig. 6). Annuals were most frequently recorded among species registered on fallows (approx. 40\%) and biennials compose $15 \%$ of its flora. The spontaneously growing bush communities include only $15 \%$ of annual species. The changes in abundance of species during the successive five years of study on most of the studied biotops were insignificant. Only on fallows, the number of segetal flora decreases. Coincidentally, an increase of ruderal species and the occurrence of a bigger number of perennials were recorded. Unfortunately, the changes in the structure of fallow flora were associated with a decline in the number of flow species.

The spontaneously growing bushes most frequently develop on the edges of arable fields, the sunny slopes of loess ravines and were frequently covered by patches, different in size, predominated by Prunus fruticosa which is very important in the early spring aspect. Other shrubs often present and intensely foraged were Rosa canina, $R$. dumalis, Crataegus monogyna. Accompanying species were mainly heliophytes of the edge communities and meadow taxons: Clinopodium vulgare, Prunella vulgaris, Heracleum sphondylium, Knautia arvensis or Agrimonia eupatoria. The two last mentioned are particularly attractive for bees.

The most frequently found on field margins were Achillea millefolium, Agrimonia eupatoria, Anthriscus sylvestris, Berteroa incana, Cichorium intybus, Euphorbia cyparisias, Hypericum perforatum, Lamium album, Lotus corniculatus, Potentilla anserina, Sedum maximum, Verbascum densiflorum, Vicia cracca. The above species are valuable for pollinators as they form dense patches or are characterised by a long period of blooming. 
Table 1

Alphabetical list and characterization of species (averages from 2002 2006).

\begin{tabular}{|c|c|c|c|c|c|c|c|c|c|c|c|}
\hline \multirow{2}{*}{ A } & \multirow{2}{*}{ B } & \multirow{2}{*}{$\mathrm{C}$} & \multirow{2}{*}{$\mathrm{D}$} & \multicolumn{2}{|c|}{ E } & \multicolumn{2}{|c|}{$\mathrm{F}$} & \multicolumn{2}{|c|}{$\mathrm{G}$} & \multirow{2}{*}{$\mathrm{H}$} & \multirow{2}{*}{ I } \\
\hline & & & & $\mathrm{a}$ & $\mathrm{b}$ & $\mathrm{a}$ & $\mathrm{b}$ & $\mathrm{a}$ & $\mathrm{b}$ & & \\
\hline Achillea millefolium L. & $\mathrm{p}$ & Ap & M A & + & 2 & + & 2 & + & 1 & $20.05 \quad 30.09$ & medium \\
\hline Adonis aestivalis $\mathrm{L}$. & $\mathrm{a}$ & Arch & SM & + & 1 & . & . & . & & $10.05 \quad 10.06$ & weak \\
\hline Aegopodium podagraria $\mathrm{L}$. & $\mathrm{p}$ & Ap & Q F & . & . & + & 1 & + & 2 & $20.05 \quad 10.07$ & weak \\
\hline Agrimonia eupatoria $\mathrm{L}$. & $\mathrm{p}$ & Ap & F B & + & 2 & + & 2 & + & 2 & $\begin{array}{ll}10.06 & 15.08 \\
\end{array}$ & good \\
\hline Agrostis capillaris L. & $\mathrm{p}$ & $\mathrm{Ap}$ & $\mathrm{NC}$ & + & 2 & + & 2 & . & . & & \\
\hline Amaranthus retroflexus L. & $\mathrm{a}$ & Ep & & + & 2 & . & . & . & . & $20.07 \quad 20.09$ & weak \\
\hline Anchusa officinalis L. & $\mathrm{b}$ & Arch & $\mathrm{AR}$ & + & 1 & . & . & . & . & $15.05 \quad 30.09$ & $\mathrm{v}$ good \\
\hline Anthriscus sylvestris (L.) Hoffm. & $\mathrm{p}$ & Ap & $\mathrm{AR}$ & . & . & + & 2 & . & . & $15.05 \quad 15.06$ & weak \\
\hline Anthyllis vulneraria $\mathrm{L}$. & $\mathrm{p}$ & Ap & M A & + & 2 & + & 2 & . & - & $20.05 \quad 30.07$ & medium \\
\hline Apera spica-venti (L.) P. Beauv. & $\mathrm{a}$ & Arch & SM & + & 2 & + & 1 & + & 1 & & \\
\hline Arenaria serpyllifolia L. & $\mathrm{a}, \mathrm{b}$ & Ap & & + & 2 & . & . & . & . & $20.05 \quad 30.09$ & weak \\
\hline Armoracia rusticana Gaertn. & $\mathrm{p}$ & $\mathrm{Ap}$ & $\mathrm{AR}$ & + & 1 & + & 1 &. & . & $\begin{array}{ll}15.05 & 20.08 \\
\end{array}$ & weak \\
\hline Artemisia absinthium L. & $\mathrm{p}$ & Arch & $\mathrm{AR}$ & + & 2 & + & 2 & . & . & $10.07 \quad 30.09$ & weak \\
\hline Artemisia campestris L. & $\mathrm{p}$ & Ap & F B & + & 2 & . & . & . & . & $15.07 \quad 20.10$ & weak \\
\hline Artemisia vulgaris $\mathrm{L}$. & $\mathrm{p}$ & Ap & $\mathrm{AR}$ & + & 2 & + & 1 &. & . & $15.07 \quad 20.10$ & weak \\
\hline Astragalus cicer $\mathrm{L}$. & $\mathrm{p}$ & Ap & TG & + & 2 & . & . & . & . & $15.05 \quad 20.07$ & medium \\
\hline Ballota nigra $\mathrm{L}$. & $\mathrm{p}$ & Arch & $\mathrm{AR}$ & . & . & . & . & + & 2 & $01.07 \quad 10.09$ & v good \\
\hline Bellis perennis $\mathrm{L}$. & $\mathrm{p}$ & Ap & $\mathrm{M} \mathrm{A}$ & + & 2 & + & 2 & . & $\cdot$ & $20.04 \quad 15.07$ & weak \\
\hline Berteroa incana (L.) DC. & $\mathrm{a}$ & Ap & $\mathrm{AR}$ & + & 2 & + & 2 & . & . & $10.05 \quad 30.09$ & v good \\
\hline Bromus hordeaceus L. & $\mathrm{b}$ & Ap & $\mathrm{M} \mathrm{A}$ &. &. & + & 1 &. & . & & \\
\hline Bromus inermis Leyss. & $\mathrm{p}$ & Ap & AIR & . & . & + & 1 & . & . & & \\
\hline Bunias orientalis L. & $\mathrm{b}$ & Ep & & . & . & . & . & . & . & $05.05 \quad 10.06$ & v good \\
\hline Campanula glomerata $\mathrm{L}$. & $\mathrm{p}$ & Ap & F B & + & 1 & + & 1 & . & . & $10.06 \quad 01.09$ & medium \\
\hline Campanula patula $\mathrm{L}$. & $\mathrm{b}, \mathrm{p}$ & Ap & M A & + & 1 & + & 1 & . & . & $20.05 \quad 10.07$ & v good \\
\hline Campanula rapunculoides $\mathrm{L}$. & $\mathrm{p}$ & $\mathrm{Ap}$ & TG & + & 1 & + & 1 & . & . & $\begin{array}{ll}10.06 & 01.09 \\
\end{array}$ & v good \\
\hline Campanula rotundifolia $\mathrm{L}$. & $\mathrm{p}$ & Ap & & + & 1 & . & . & . & . & $10.06 \quad 01.09$ & v good \\
\hline Capsella bursa-pastoris (L.) Med. & $\mathrm{a}$ & Ap & SM & + & 2 & + & 1 & . & . & $20.04 \quad 10.10$ & v good \\
\hline Cardaminopsis arenosa (L.) Hayek & $\mathrm{b}, \mathrm{p}$ & Ap & & + & 2 & . & . & . & . & 20.0420 .05 & weak \\
\hline Carex hirta L. & $\mathrm{p}$ & Ap & M A & + & 2 & + & 2 & . & -1 & $10.05 \quad 20.06$ & medium \\
\hline Carlina vulgaris $\mathrm{L}$. & $\mathrm{b}$ & Ap & F B & + & 1 &. &. &. & . & $20.07 \quad 15.08$ & good \\
\hline Centaurea cyanus L. & $\mathrm{a}$ & Arch & SM & + & 1 & . & . & . & . & $10.06 \quad 01.08$ & $\mathrm{v}$ good \\
\hline Centaurea jacea $\mathrm{L}$. & $\mathrm{p}$ & Ap & M A & + & 1 & + & 1 & . & . & $20.06 \quad 20.08$ & v good \\
\hline Centaurea pannonica (Heuff.) Hayek & $\mathrm{p}$ & Ap & F B & . & . & + & 1 & . & . & $20.06 \quad 20.08$ & v good \\
\hline Centaurea scabiosa $\mathrm{L}$. & $\mathrm{p}$ & Ap & F B & + & 2 & + & 1 & . & . & $20.06 \quad 10.09$ & v good \\
\hline Centaurea stoebe L. & $\mathrm{b}$ & Ap & F B & + & 1 & . & . & . & . & $\begin{array}{ll}25.06 \quad 20.08 \\
\end{array}$ & v good \\
\hline Centaurium erythraea Rafn & $\mathrm{b}$ & Ap & IN & + & 2 & . & . & . & . & $30.06 \quad 30.08$ & v good \\
\hline Cerastium arvense L.s.s. & $\mathrm{p}$ & Ap & & + & 2 & + & 1 & . & . & $10.05 \quad 30.06$ & medium \\
\hline Cerastium holosteoides Fr. Emend.Hyl. & $\mathrm{a}$ & Ap & M A & + & 2 & . & . & . & - & $10.05 \quad 30.07$ & weak \\
\hline Chamaecytisus ratisbonensis (Schaeff.) Rothm. & $\mathrm{s}$ & Ap & & + & 2 & + & 2 & + & 2 & $10.05 \quad 15.06$ & weak \\
\hline Chamaenerion angustifolium (L.) Scop. & $\mathrm{p}$ & Ap & EP & . &. & + & 1 & . & . & 20.0620 .07 & v good \\
\hline Chamomilla suaveolens (Pursh) Rydb. & $\mathrm{a}$ & Ep & M A & + & 2 & . & . & . & . & $10.06 \quad 20.07$ & v good \\
\hline Chelidonium majus L. & $\mathrm{p}$ & Ap & $\mathrm{AR}$ &. &. & + & 1 & + & 2 & $\begin{array}{ll}05.05 & 10.10 \\
\end{array}$ & good \\
\hline Chenopodium album L. & $\mathrm{a}$ & Ap & SM & + & 1 & + & 1 & . & . & $20.06 \quad 20.09$ & good \\
\hline Cichorium intybus L. & $\mathrm{p}$ & Arch & $\mathrm{AR}$ & + & 2 & + & 2 & . & . & $10.06 \quad 01.09$ & v.good \\
\hline Cirsium arvense (L.) Scop. & $\mathrm{p}$ & Ap & $\mathrm{AR}$ & + & 2 & + & 1 & . & . & $\begin{array}{ll}30.06 \quad 20.08 \\
\end{array}$ & v good \\
\hline Clinopodium vulgare $\mathrm{L}$. & $\mathrm{p}$ & Ap & TG & . & . & + & 2 & + & 1 & $15.07 \quad 20.08$ & good \\
\hline Consolida regalis Gray & $\mathrm{a}$ & Arch & SM & + & 1 & + & 1 & . & $\cdot$ & $10.06 \quad 20.07$ & medium \\
\hline Convolvulus arvensis $\mathrm{L}$. & $\mathrm{p}$ & Ap & AIR & + & 2 & + & 2 & . & . & $10.06 \quad 10.09$ & medium \\
\hline Conyza canadensis $(\mathrm{L}$.$) Conquist$ & $\mathrm{a}$ & Ep & SM & + & 2 & + & 2 & . & . & & \\
\hline Coronilla varia $\mathrm{L}$. & $\mathrm{p}$ & Ap & TG & + & 1 & + & 2 & . & $\bar{c}$ & $10.06 \quad 10.08$ & good \\
\hline Crataegus monogyna Jacq. & $\mathrm{s} / \mathrm{t}$ & Ap & $\mathrm{RP}$ & + & 1 & + & 1 & + & 2 & $15.05 \quad 30.05$ & v good \\
\hline Crepis tectorum $\mathrm{L}$. & $\mathrm{a}, \mathrm{b}$ & Ap & SM &. &. & + & 1 &. & $\cdot$ & $10.06 \quad 10.09$ & mebium \\
\hline Dactylis glomerata $\mathrm{L}$. & $\mathrm{p}$ & Ap & $\mathrm{M} \mathrm{A}$ & + & 2 & + & 2 & . & $\dot{.}$ & & \\
\hline Daucus carota $\mathrm{L}$. & $\mathrm{b}$ & Ap & M A & + & 2 & + & 2 & . & $\cdot$ & $20.06 \quad 15.09$ & weak \\
\hline Descurainia sophia $(\mathrm{L}$.)Webb ex Prantl & $\mathrm{a} / \mathrm{b}$ & Arch & SM & + & 1 & + & 1 & . & $\cdot$ & $\begin{array}{ll}20.05 & 10.10 \\
\end{array}$ & weak \\
\hline Dianthus carthusianorum L. & $\mathrm{p}$ & Ap & F B & + & 2 & . & . & . & . & $15.06 \quad 20.07$ & good \\
\hline Dianthus delthoides $\mathrm{L}$. & $\mathrm{p}$ & Ap & KG & + & 2 & + & 2 & & & $20.06 \quad 20.07$ & weak \\
\hline
\end{tabular}


cd. Table 1

\begin{tabular}{|c|c|c|c|c|c|c|c|c|c|c|c|}
\hline \multirow{2}{*}{ A } & \multirow{2}{*}{ B } & \multirow{2}{*}{$\mathrm{C}$} & \multirow{2}{*}{$\mathrm{D}$} & \multicolumn{2}{|c|}{ E } & \multicolumn{2}{|c|}{$\mathrm{F}$} & \multicolumn{2}{|c|}{$\mathrm{G}$} & \multirow{2}{*}{$\mathrm{H}$} & \multirow{2}{*}{ I } \\
\hline & & & & $\mathrm{a}$ & $\mathrm{b}$ & $\mathrm{a}$ & $\mathrm{b}$ & $\mathrm{a}$ & $\mathrm{b}$ & & \\
\hline Echinocystis lobata (F.Michx.)Torr. \& Agray & $\mathrm{a}$ & $\mathrm{Ag}$ & & . & . & . & 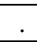 & + & 2 & $\begin{array}{ll}30.06 & 15.08 \\
\end{array}$ & weak \\
\hline Echium vulgare $\mathrm{L}$. & $\mathrm{b}$ & Ap & AR & + & 2 & + & 1 & . & . & $10.06 \quad 10.09$ & v good \\
\hline Elymus repens (L.) Gould. & $\mathrm{p}$ & $\mathrm{Ap}$ & AIR & + & 2 & + & 2 & 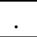 & - & & \\
\hline Epilobium montanum $\mathrm{L}$. & $\mathrm{p}$ & Ap & $\mathrm{AR}$ & . & . & + & 1 & + & 1 & $20.06 \quad 30.08$ & $\mathrm{v}$ good \\
\hline Equisetum arvense L. & $\mathrm{p}$ & Ap & AIR & + & 3 & + & 2 & . & 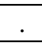 & & \\
\hline Erigeron annuus (L.)Pers. & $\mathrm{b} / \mathrm{p}$ & $\mathrm{Ag}$ & & + & 2 & + & 2 & . & . & $20.06 \quad 20.09$ & weak \\
\hline Erigeron acris $\mathrm{L}$. & $\mathrm{b} / \mathrm{p}$ & Ap & & + & 1 & . & & . & . & $20.06 \quad 20.10$ & weak \\
\hline Erodium cicutarium (L.) L Her. & $\mathrm{a}$ & Ap & & + & 1 & . & & 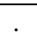 & & $20.05 \quad 20.07$ & weak \\
\hline Euоnутия europea L. & $\mathrm{s}$ & Ap & $\mathrm{RP}$ & . & 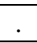 & . & & + & 2 & $\begin{array}{ll}15.05 \quad 10.06 \\
\end{array}$ & good \\
\hline Euphorbia cyparissias L. & $\mathrm{p}$ & Ap & F B & + & 1 & + & 3 & . & . & $01.05 \quad 20.05$ & good \\
\hline Euphorbia esula $\mathrm{L}$. & $\mathrm{p}$ & Ap & & + & 2 & . & 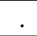 & . & . & $20.05 \quad 20.07$ & good \\
\hline Euphorbia helioscopia L. & $\mathrm{a}$ & Arch & SM & + & 1 & & & . & . & $10.05 \quad 10.06$ & weak \\
\hline Euphrasia rostkoviana Hayne & $\mathrm{p}$ & Ap & M A & + & 1 & . & & . & . & $\begin{array}{ll}15.07 & 15.09\end{array}$ & weak \\
\hline Falcaria vulgaris Bernh. & $\mathrm{b} / \mathrm{p}$ & Ap & AIR & . & . & + & 2 & . & . & $20.06 \quad 15.08$ & weak \\
\hline Fallopia convolvulus (L.) A.Love & $\mathrm{a}$ & Arch & SM & + & 2 & . & . & . & . & $20.05 \quad 20.06$ & medium \\
\hline Filipendula vulgaris Moench & $\mathrm{p}$ & $\mathrm{Ap}$ & F B & + & 2 & . & & . & . & $20.06 \quad 10.08$ & v good \\
\hline Fragaria vesca $\mathrm{L}$. & $\mathrm{p}$ & Ap & EP & . & & + & 3 & . & . & $\begin{array}{ll}10.05 & 10.06\end{array}$ & medium \\
\hline Fumaria officinalis $\mathrm{L}$. & $\mathrm{a}$ & Arch & SM & + & 2 & . & & . & . & $20.05 \quad 20.08$ & weak \\
\hline Gagea pratensis (Pers.)Dum. & $\mathrm{p}$ & Ap & SM & . & . & + & 2 & . & . & $15.04 \quad 20.05$ & good \\
\hline Galeopsis pubescens Besser & $\mathrm{a}$ & Ap & $\mathrm{AR}$ & + & 1 & + & 1 & . & . & $\begin{array}{ll}15.06 & 01.09\end{array}$ & v good \\
\hline Galeopsis tetrahit $\mathrm{L}$. & $\mathrm{a}$ & Ap & SM & . & & . & & + & 1 & $10.06 \quad 20.09$ & medium \\
\hline Galinsoga ciliata (Raf.)S.F.Blake & $\mathrm{a}$ & Ep & SM & + & 2 & . & & . & & $15.07 \quad 30.09$ & weak \\
\hline Galinsoga parviflora Cav. & $\mathrm{a}$ & Ep & SM & + & 2 & . & . & . & . & $\begin{array}{ll}15.07 \quad 30.10 \\
\end{array}$ & medium \\
\hline Galium aparine L. & $\mathrm{a}$ & Ap & $\mathrm{AR}$ & + & 1 & + & 1 & + & 1 & $\begin{array}{ll}10.06 & 15.09\end{array}$ & weak \\
\hline Galium mollugo L. & $\mathrm{p}$ & Ap & $\mathrm{M} \mathrm{A}$ & + & 1 & + & 2 & + & 1 & $\begin{array}{ll}15.06 & 15.09 \\
\end{array}$ & weak \\
\hline Galium verum L. & $\mathrm{p}$ & Ap & TG & . & . & + & 2 & . & . & $10.07 \quad 20.09$ & medium \\
\hline Geranium pratense L. & $\mathrm{p}$ & Ap & M A & + & 1 & + & 1 & . & . & $10.06 \quad 10.08$ & medium \\
\hline Geranium pusillum Burm. F. ex L. & $\mathrm{a}$ & Arch & SM & + & 1 & & & . & . & $15.06 \quad 20.08$ & weak \\
\hline Geranium robertianum L. & $\mathrm{a} / \mathrm{b}$ & Ap & $\mathrm{AR}$ & + & 1 & . & . & . & . & $\begin{array}{ll}15.05 \quad 20.07 \\
\end{array}$ & medium \\
\hline Geum urbanum L. & $\mathrm{p}$ & Ap & $\mathrm{AR}$ & . & . & + & 1 & + & 1 & $01.06 \quad 20.06$ & good \\
\hline Glechoma hederacea $\mathrm{L}$. & $\mathrm{p}$ & Ap & AR & . & . & + & 1 & + & 2 & $20.04 \quad 10.07$ & good \\
\hline Gypsophilla muralis L. & $\mathrm{a}$ & Ap & IN & + & 2 & 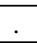 & & . &. & & \\
\hline Helichrysum arenarium (L.) Moench & $\mathrm{b}$ & Ap & KG & + & 2 & + & 2 & & & $15.07 \quad 20.08$ & weak \\
\hline Heracleum sibiricum L. & $\mathrm{b} / \mathrm{p}$ & Ap & M A & . & . & + & 1 & . & . & $10.07 \quad 5.08$ & weak \\
\hline Heracleum sphondylium L. & $\mathrm{b} / \mathrm{p}$ & Ap & M A & + & 1 & + & 1 & . & . & $\begin{array}{ll}15.06 & 1.09\end{array}$ & weak \\
\hline Herniaria glabra $\mathrm{L}$. & $\mathrm{a} / \mathrm{b}$ & Ap & KG & . & . & . & & . & . & & \\
\hline Hieracium bauhinii Schult. & $\mathrm{p}$ & Ap & F B & + & 2 & - & & . & . & $\begin{array}{ll}25.06 \quad 20.07 \\
\end{array}$ & weak \\
\hline Hieracium pilosella L. & $\mathrm{p}$ & Ap & $\mathrm{NC}$ & + & 2 & + & 2 & . & . & $20.05 \quad 30.07$ & good \\
\hline Hieracium umbellatum $\mathrm{L}$. & $\mathrm{p}$ & Ap & $\mathrm{NC}$ & + & 2 & . & . & . & . & $\begin{array}{ll}30.07 & 10.10 \\
\end{array}$ & good \\
\hline Hypericum perforatum L. & $\mathrm{p}$ & Ap & & + & 2 & + & 2 & . & . & $05.06 \quad 30.07$ & v good \\
\hline Jasione montana $\mathrm{L}$. & $\mathrm{b}$ & Ap & KG & + & 2 & . & & . & . & $\begin{array}{ll}10.06 \quad 30.07 \\
\end{array}$ & medium \\
\hline Knautia arvensis (L.) J. M. Coult. & $\mathrm{p}$ & Ap & M A & + & 1 & + & 2 & . & . & $\begin{array}{ll}10.06 \quad 30.07 \\
\end{array}$ & v good \\
\hline Lactuca seriola $\mathrm{L}$. & $\mathrm{b}$ & Arch & SM & + & 1 & + & 1 & . & . & $20.06 \quad 20.09$ & weak \\
\hline Lamium album L. & $\mathrm{p}$ & Arch & $\mathrm{AR}$ & + & 1 & + & 1 & + & 1 & $20.04 \quad 30.09$ & v good \\
\hline Lamium amplexicaule L. & $\mathrm{a}$ & Arch & SM & + & 1 & . & . & . & . & $01.04 \quad 30.06$ & good \\
\hline Lamium purpureum L. & $\mathrm{a}$ & Arch & SM & + & 1 & 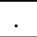 & & . & . & $\begin{array}{ll}15.04 & 01.09\end{array}$ & v good \\
\hline Lapsana communis L.s.s. & $\mathrm{a}$ & $\mathrm{Ap}$ & SM & + & 1 & + & 1 & . & . & $20.06 \quad 20.09$ & medium \\
\hline Lathyrus pratensis $\mathrm{L}$. & $\mathrm{p}$ & Ap & $\mathrm{M} \mathrm{A}$ & + & 2 & + & 2 & . & . & $\begin{array}{ll}15.06 & 15.07 \\
\end{array}$ & good \\
\hline Lavathera turingiaca & $\mathrm{b}$ & Ap & & & & + & 1 & & & $\begin{array}{ll}15.07 & 15.08\end{array}$ & medium \\
\hline Leontodon autumnalis $\mathrm{L}$. & $\mathrm{p}$ & Ap & M A & + & 1 & + & 2 & . & . & $20.06 \quad 30.09$ & v good \\
\hline Leontodon hispidus L. & $\mathrm{p}$ & Ap & $\mathrm{M} \mathrm{A}$ & . & . & + & 1 & . & . & $\begin{array}{ll}30.06 & 15.10\end{array}$ & weak \\
\hline Lepidium ruderale L. & $\mathrm{a}$ & Ap & SM & + & 1 & . & . & . & . & $20.05 \quad 10.09$ & weak \\
\hline Leucanthemum vulgare & $\mathrm{p}$ & Ap & $\mathrm{M} \mathrm{A}$ & + & 2 & & & & & $\begin{array}{ll}15.06 & 15.08 \\
\end{array}$ & weak \\
\hline Linaria vulgaris Mill. & $\mathrm{p}$ & Ap & $\mathrm{AR}$ & + & 1 & + & 1 & & & $\begin{array}{ll}15.06 \quad 20.09 \\
\end{array}$ & v good \\
\hline Lithospermum arvense $\mathrm{L}$. & $\mathrm{a} / \mathrm{b}$ & Arch & SM & + & 1 & . & & . & . & $\begin{array}{ll}15.06 & 20.07\end{array}$ & weak \\
\hline Lolium perenne $\mathrm{L}$. & $\mathrm{p}$ & Ap & M A & + & 2 & + & 2 & . & . & & \\
\hline Lotus corniculatus L. & $\mathrm{p}$ & Ap & $\mathrm{M} \mathrm{A}$ & + & 2 & + & 2 & + & 1 & $\begin{array}{ll}10.05 & 15.09 \\
\end{array}$ & v good \\
\hline Luzula campestris (L.) DC. & $\mathrm{p}$ & Ap & $\mathrm{NC}$ & + & 1 & & & & & & \\
\hline Lychnis flos-cuculi L. & $\mathrm{p}$ & Ap & $\mathrm{M} \mathrm{A}$ & + & 1 & + & 1 & . & . & $\begin{array}{ll}10.05 \quad 25.06 \\
\end{array}$ & v good \\
\hline
\end{tabular}


cd. Table 1

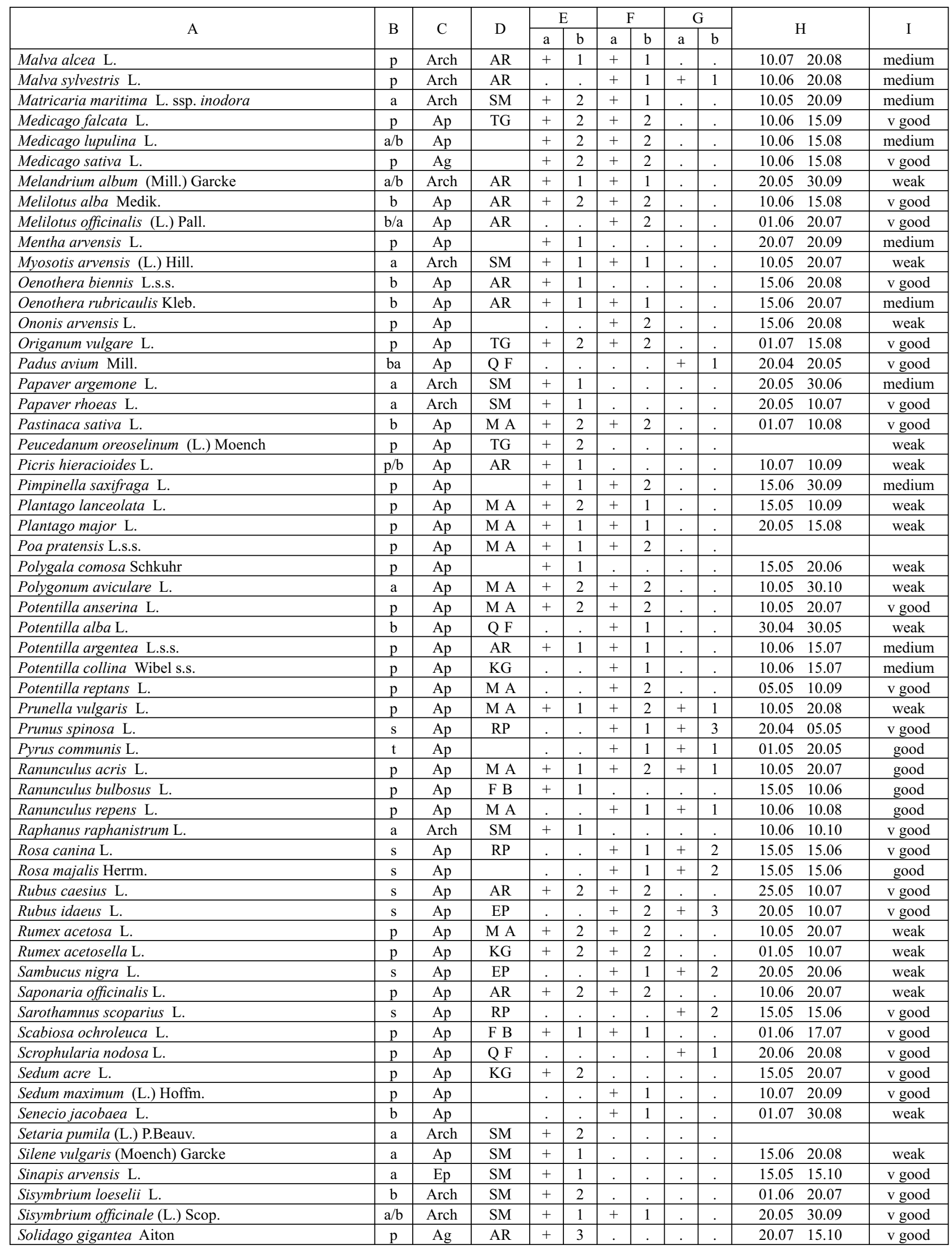


cd. Table 1

\begin{tabular}{|c|c|c|c|c|c|c|c|c|c|c|c|}
\hline \multirow{2}{*}{ A } & \multirow{2}{*}{ B } & \multirow{2}{*}{$\mathrm{C}$} & \multirow{2}{*}{$\mathrm{D}$} & \multicolumn{2}{|c|}{ E } & \multicolumn{2}{|c|}{$\mathrm{F}$} & \multicolumn{2}{|c|}{$\mathrm{G}$} & \multirow{2}{*}{$\mathrm{H}$} & \multirow{2}{*}{ I } \\
\hline & & & & $\mathrm{a}$ & $\mathrm{b}$ & $\mathrm{a}$ & $\mathrm{b}$ & $\mathrm{a}$ & $\mathrm{b}$ & & \\
\hline Sonchus arvensis L. & $\mathrm{p}$ & Ap & SM & + & 1 & + & 1 & & & $28.07 \quad 10.10$ & weak \\
\hline Sonchus oleraceus L. & $\mathrm{a}$ & Arch & SM & + & 1 & . & . & . & & $20.06 \quad 15.09$ & weak \\
\hline Sorbus aucuparia L. & $\mathrm{t}$ & $\mathrm{Ap}$ & & . & & & & + & 1 & $\begin{array}{ll}10.05 \quad 30.05 \\
\end{array}$ & medium \\
\hline Stachys palustris L. & $\mathrm{p}$ & Ap & $\mathrm{M} \mathrm{A}$ & + & 1 & . & . & . & . & $\begin{array}{ll}10.06 \quad 20.09 \\
\end{array}$ & medium \\
\hline Stellaria graminea $\mathrm{L}$. & $\mathrm{p}$ & Ap & & . & & + & 1 & . & & $\begin{array}{ll}10.05 \quad 20.06 \\
\end{array}$ & weak \\
\hline Stellaria media (L.) Vill. & $\mathrm{a}$ & Ap & SM & + & 2 & & & 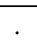 & & $\begin{array}{ll}20.03 & 30.10\end{array}$ & weak \\
\hline Symphytum officinale L. & $\mathrm{p}$ & Ap & & . & & . & $\cdot$ & + & 1 & $\begin{array}{ll}15.05 & 20.08 \\
\end{array}$ & v good \\
\hline Tanacetum vulgare $\mathrm{L}$. & $\mathrm{p}$ & Ap & $\mathrm{AR}$ & + & 2 & + & 2 & & & $20.07 \quad 01.10$ & good \\
\hline Taraxacum officinale F. H.Wigg. & $\mathrm{p}$ & $\mathrm{Ap}$ & $\mathrm{M} \mathrm{A}$ & + & 2 & + & 2 & . & . & $05.05 \quad 25.05$ & $\mathrm{v}$ good \\
\hline Thlaspi arvense $\mathrm{L}$. & $\mathrm{a}$ & Ap & SM & + & 1 & . & . & . & - & $\begin{array}{ll}15.05 & 20.08 \\
\end{array}$ & weak \\
\hline Thymus pulegioides L. & $\mathrm{p}$ & Ap & & + & 2 & & & 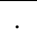 & & $10.06 \quad 10.07$ & $\mathrm{v}$ good \\
\hline Thymus serpyllum L. & $\mathrm{p}$ & Ap & KG & + & 2 & . & . & . & 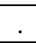 & $\begin{array}{ll}10.06 \quad 10.07 \\
\end{array}$ & $\mathrm{v}$ good \\
\hline Tilia cordata $\mathrm{L}$. & $\mathrm{t}$ & Ap & Q F & . &. & . & . & + & 1 & $\begin{array}{ll}30.06 \quad 20.07 \\
\end{array}$ & v.good \\
\hline Tragopogon pratensis L.s.s. & $\mathrm{b}$ & Ap & $\mathrm{MA}$ & . & & + & 1 & . & & $\begin{array}{ll}15.05 & 20.07\end{array}$ & medium \\
\hline Trifolium alpestre $\mathrm{L}$. & $\mathrm{b}$ & Ap & TG & . & . & + & 1 & . & . & $20.06 \quad 15.07$ & medium \\
\hline Trifolium arvense $\mathrm{L}$. & $\mathrm{a} / \mathrm{b}$ & Ap & $\mathrm{KG}$ & + & 2 & + & 2 & & & $01.06 \quad 30.07$ & v good \\
\hline Trifolium campestre Schreb. & $\mathrm{a} / \mathrm{b}$ & Ap & KG & + & 1 & . & . & . & . & $\begin{array}{ll}01.06 \quad 30.07 \\
\end{array}$ & medium \\
\hline Trifolium dubium Sibth. & $\mathrm{a} / \mathrm{b}$ & Ap & $\mathrm{M} \mathrm{A}$ & + & 1 & . & - & . & 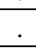 & $01.06 \quad 30.07$ & $\mathrm{v}$ good \\
\hline Trifolium hybridum L. & $\mathrm{p}$ & Ap & $\mathrm{M} \mathrm{A}$ & . & & + & 2 & & & $\begin{array}{ll}01.06 \quad 30.07 \\
\end{array}$ & v good \\
\hline Trifolium medium L. & $\mathrm{p}$ & Ap & TG & . & . & + & 2 & . & . & $01.06 \quad 30.07$ & v good \\
\hline Trifolium pratense L. & $\mathrm{p}$ & Ap & $\mathrm{M} \mathrm{A}$ & + & 1 & + & 1 & - & & $\begin{array}{ll}01.06 \quad 30.07 \\
\end{array}$ & $\mathrm{v}$ good \\
\hline $\begin{array}{l}\text { Trifolium repens } \mathrm{L} \text {. } \\
\text { The }\end{array}$ & $\mathrm{p}$ & Ap & M A & + & 2 & + & 2 & . & & $20.05 \quad 30.08$ & $\mathrm{v}$ good \\
\hline Tussilago farfara $\mathrm{L}$. & $\mathrm{p}$ & Ap & AIR & + & 2 & + & 2 & . & . & $01.04 \quad 25.04$ & $\mathrm{v}$ good \\
\hline Urtica dioica $\mathrm{L}$. & $\mathrm{p}$ & Ap & $\mathrm{AR}$ & + & 2 & + & 1 & & & & \\
\hline Verbascum densiflorum Bertol. & $\mathrm{b}$ & Ap & $\mathrm{AR}$ & . & & + & 1 & . & & $\begin{array}{ll}10.06 & 05.09 \\
\end{array}$ & medium \\
\hline Verbascum nigrum $\mathrm{L}$. & $\mathrm{b}$ & Ap & EP & . & & + & 1 & & & $\begin{array}{ll}05.07 & 10.08\end{array}$ & v good \\
\hline Verbascum phlomoides L. & $\mathrm{b}$ & Ap & $\mathrm{AR}$ & + & 1 & + & 1 & . & . & $\begin{array}{ll}10.06 & 5.09\end{array}$ & v good \\
\hline Veronica agrestis $\mathrm{L}$. & $\mathrm{a}$ & Ap & & + & 2 & & & & & $\begin{array}{ll}10.04 \quad 20.09 \\
\end{array}$ & weak \\
\hline Veronica chamaedrys L.s.s. & $\mathrm{p}$ & Ap & & + & 1 & + & 1 & & & $15.05 \quad 20.08$ & weak \\
\hline Viburnum opulus L. & $\mathrm{s}$ & Ap & RP & -1 & & + & 1 & + & 1 & $\begin{array}{ll}10.05 & 10.06 \\
\end{array}$ & v good \\
\hline Vicia angustifolia L. & $\mathrm{a}$ & Arch & SM & + & 1 & + & 1 & & & $10.05 \quad 15.08$ & medium \\
\hline Vicia cracca L. & $\mathrm{p}$ & $\mathrm{Ap}$ & $\mathrm{M} \mathrm{A}$ & + & 2 & + & 2 & . & . & $10.06 \quad 20.08$ & $\mathrm{v}$ good \\
\hline Vicia hirsuta (L.) S.F.Gray & $\mathrm{a}$ & Arch & SM & + & 1 & & & $\cdot$ & & $\begin{array}{ll}10.05 & 15.07\end{array}$ & v good \\
\hline Vicia sepium L. & $\mathrm{p}$ & Ap & TG & + & 1 & & & & & $\begin{array}{ll}10.05 \quad 30.07 \\
\end{array}$ & v good \\
\hline Vicia tetrasperma (L.) Schreb. & $\mathrm{a}$ & Arch & SM & + & 1 & . & $\cdot$ & & & $\begin{array}{ll}10.05 & 15.08 \\
\end{array}$ & $\mathrm{v}$ good \\
\hline Vicia villosa Roth. & $\mathrm{a} / \mathrm{b}$ & Arch & SM & + & 2 & + & 2 & & & $\begin{array}{ll}10.06 & 15.07\end{array}$ & v good \\
\hline Viola arvensis Murray & $\mathrm{a}$ & Arch & SM & + & 2 & & 1 & & & $\begin{array}{ll}10.05 \quad 30.09 \\
\end{array}$ & medium \\
\hline
\end{tabular}

Explanations: A species; B life span: a annual, b biennial, $\mathrm{p}$ perennial, $\mathrm{s}$ shrub, $\mathrm{t}$ tree; $\mathrm{C}$ historical and geographical groups: Ap apophytes, Arch archaeophytes, Ep epecophytes, Ag agriophytes; D phytosociological unit: AR Artemisietea vulgaris, AIR Agropyretea intermedio repentis, EP Epilobietea angustifolii, F B Festuco Brometea, IN Isoeto Nanojuncetea, KG Koelerio glaucae Corynephoretea canescentis, M A Molinio Arrhenatheretea, NC Nardo Callunetea, Q F Querco Fagetae, RP Rhamno Prunetea, SM Stelarietea mediae, TG Trifolio Geranietea sanguinei; E fallows, $\mathrm{F}$ boundary strips, $\mathrm{G}$ bush communities (a presence, $\mathrm{b}$ degree of density: 1 single, 2 loose patches, 3 dense patches); $\mathrm{H}$ average time of blooming; I Intensity of insect's visits 


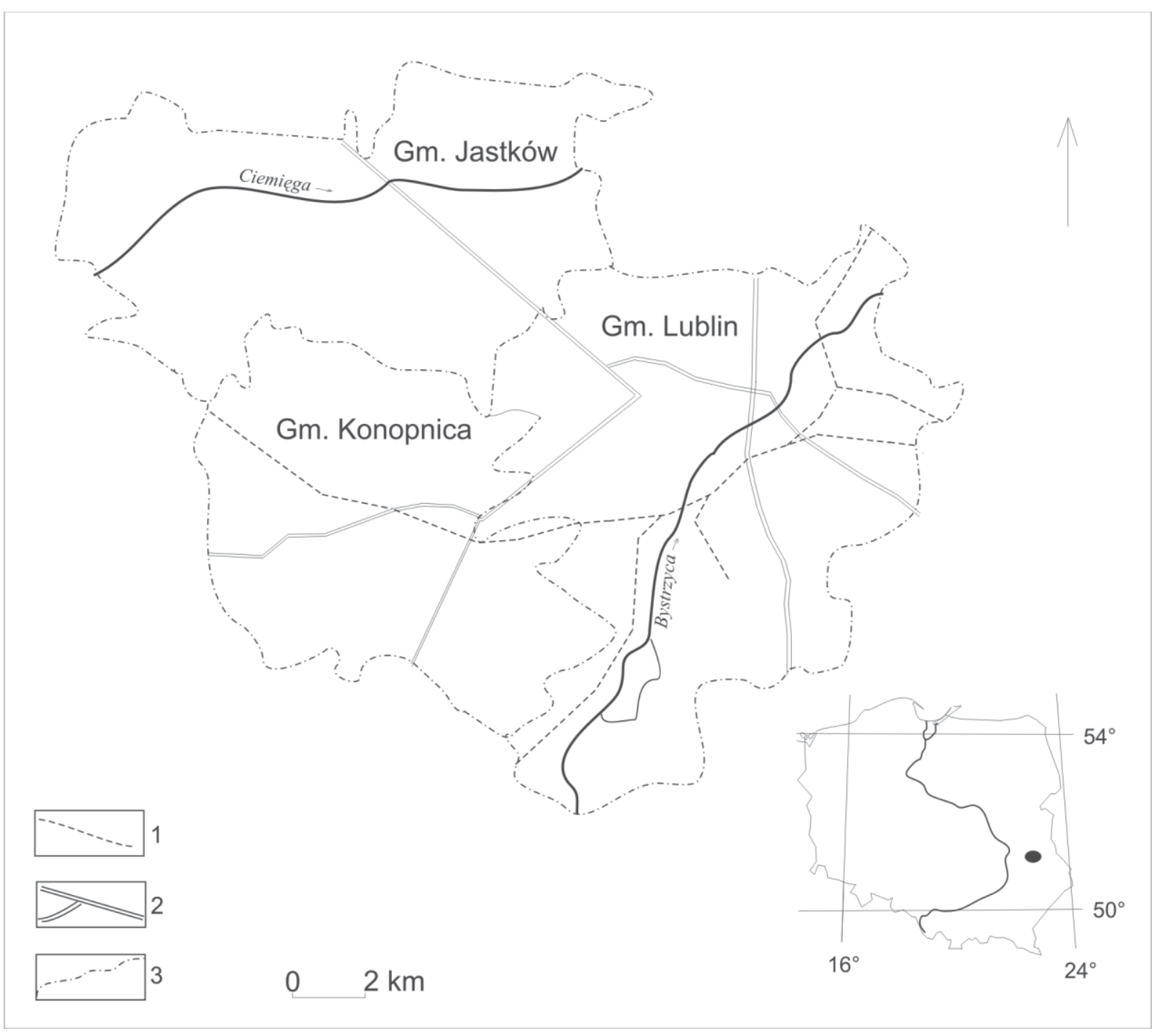

Fig. 1. The study area: 1 railway lines, 2 roads, 3 terrritorial borders.

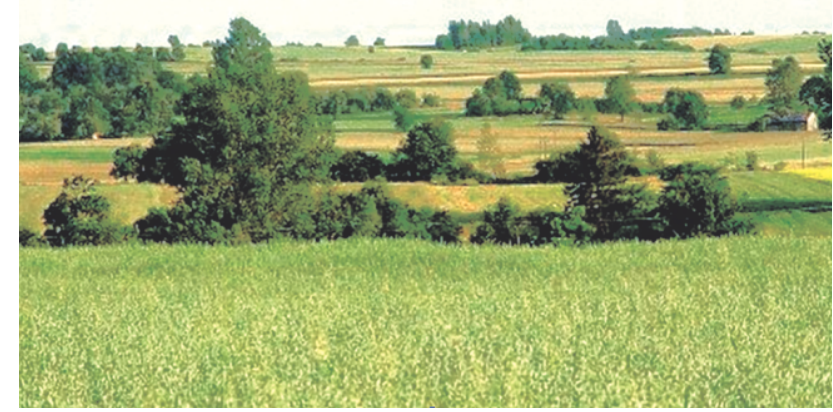

Fig. 2. Bush communities in agricultural landscape in Konopnica.

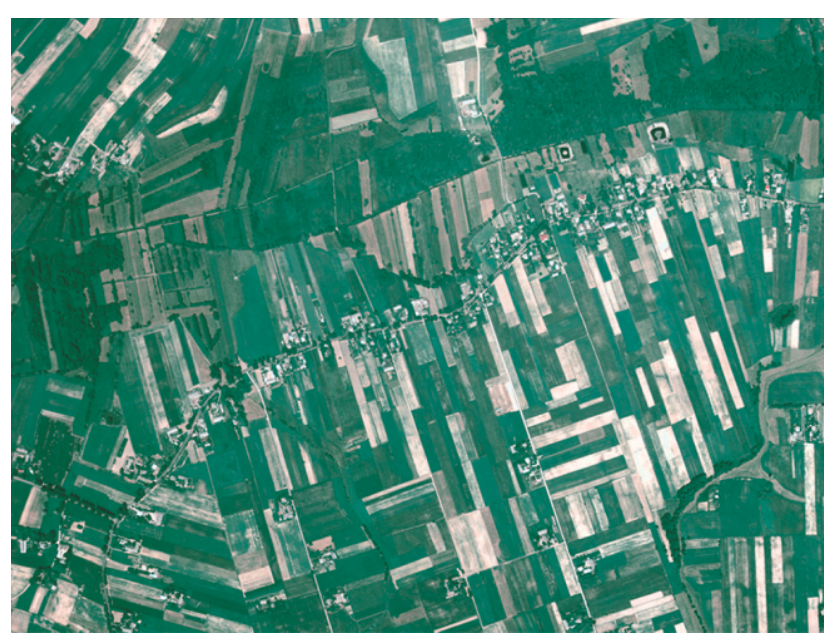

Fig. 3. Air photo showing mosaic structure of Jastków landscape. 


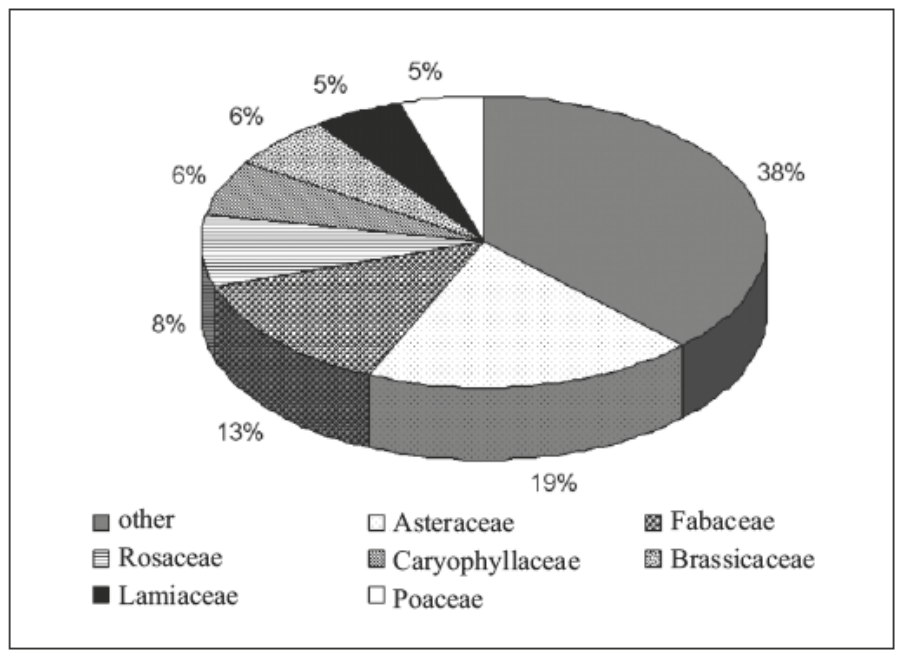

Fig. 4. The share of recorded species in botanical families.

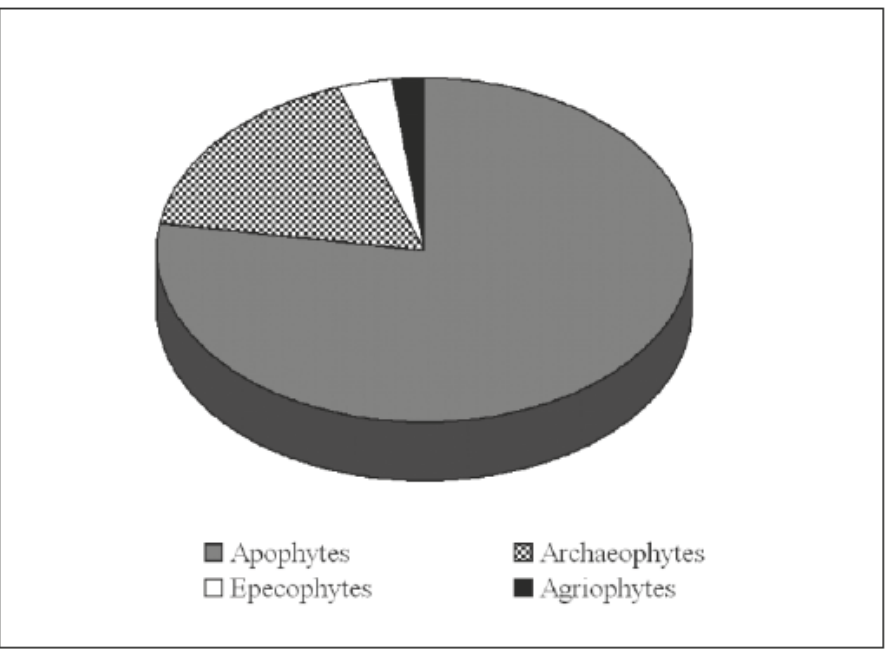

Fig. 5. The participation of geographical historical groups in recorded flora.

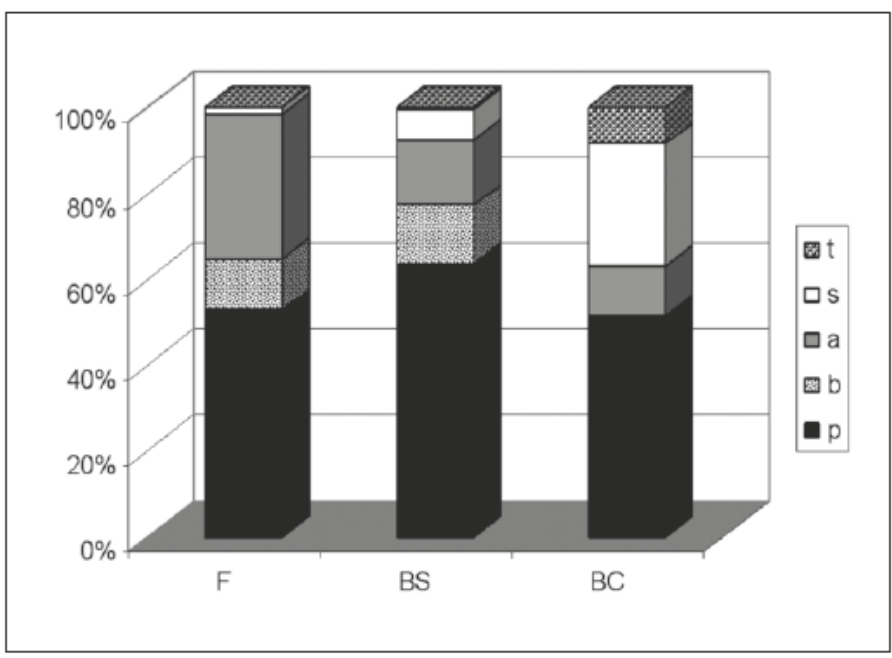

Fig. 6. Spectrum of life forms in different biotops: F: fallows, BS bus $\mathrm{h}$ communities, $\mathrm{BC}$ boundary strips, $\mathrm{t}$ tree, $\mathrm{s}$ shrubs, a annual, b biennial, p perennial. 

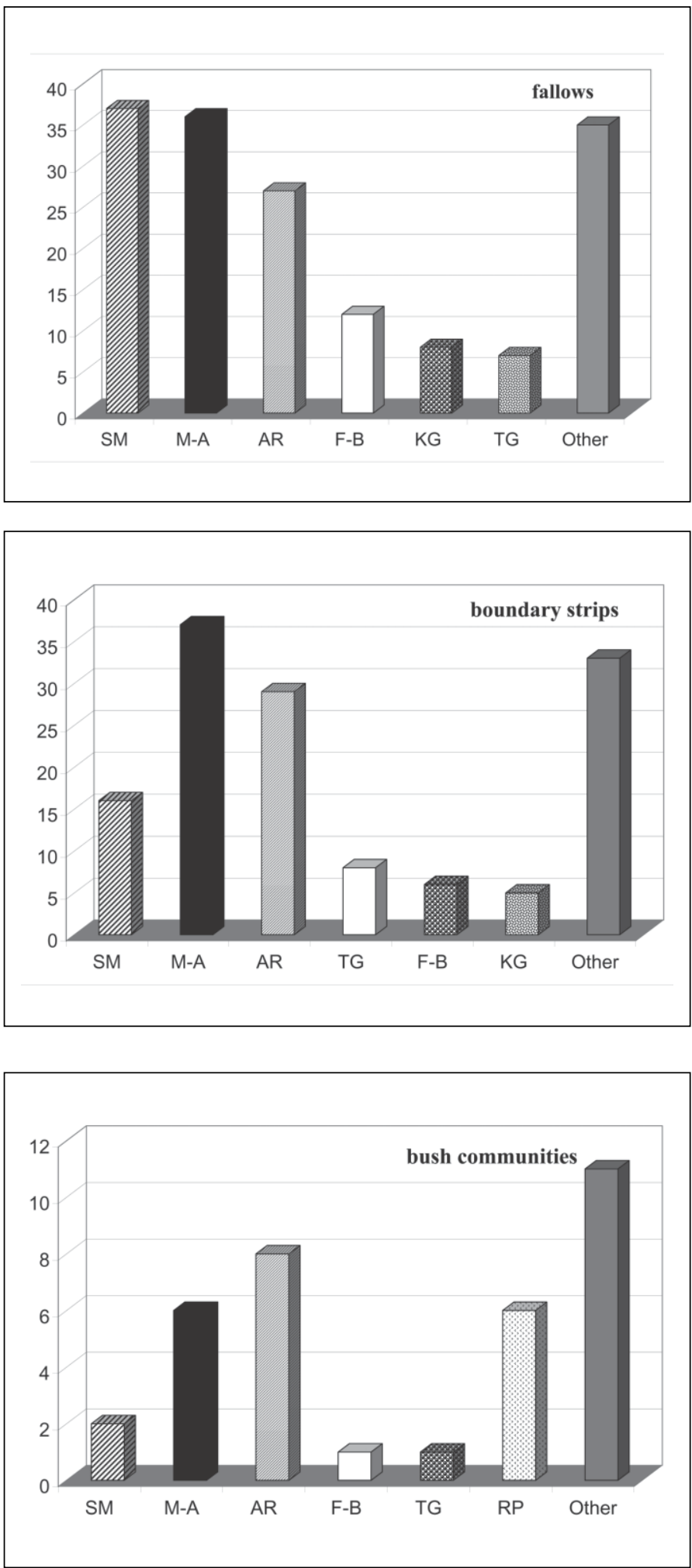

Fig. 7. Number of species in socio ecological groups AR Artemisietea vulgaris, AIR Agropyretea intermedio repentis, EP Epilobietea angustifolii, F B Festuco Brometea, IN Isoeto Nanojuncetea, KG Koelerio glaucae Corynephoretea canescentis, M A Molinio Arrhenatheretea, NC Nardo Callunetea, Q F Querco Fagetae, RP Rhamno Prunetea, SM Stelarietea mediae, TG Trifolio Geranietea sanguinei. 
Taking into consideration the sinecological group, a considerable participation of species from Stelarietea-medie and Molinio-Arrhenatheretea classes on fallows was proved (Fig. 7). On boundary strips, a decrease of segetal species from Stelarietea-medie was recorded. The share of meadow and ruderal taxons on both fallows and boundary strips was comparable. Whereas in the flora of bushes ruderal (Artemisietea vulgaris class) and thermophilous species (Rhamno-Prunetea class) predominated.

Season-long succession of bloom was observed on boundary strips and fallows. The species in bush communities bloom mainly in early spring and provide the first and rather abundant nectar and pollen flow. Our observations have shown that the blooming plants on boundary strips and fallows create favourable conditions both for feeding and nesting for different Apoidea. Apart from Apis mellifera, bumblebees and solitary bees (mainly representatives of Andrena) were present. Generally, the density of Apoidea changed during the vegetation season and correlated with the blooming spectrum on the observed biotops. The largest density in bush communities occurred in April, whereas on field margins and fallows in summer.

The presently recorded flora of anthropogenic biotops is composed of some rare species: Centaurium erythraea, Dianthus cartusianorum, Helichrysum arenarium, Lavanthera turingiaca, Ononis arvensis, Potentilla alba, Trifolium alpestre, or Viburnum opulus. That is the confirmation of great importance of such habitats as essential areas retaining floristic biodiversity.

\section{CONCLUSIONS}

1. The flora of anthropogenic refuge areas in the studied landscape comprises 214 species of which $80 \%$ were classified as nectariferous or polleniferous. Fallows and boundary strips were the most abundant in species. The majority of taxons are apophytes which predominate over antropophytes. The complete absence of efemerophytes was associated with the character of the area.

2. The great majority of flow taxons are meadow, segetal or ruderal plants. Most of nectariferous or polleniferous taxons create loose patches, a small number forms dense areas, but the time and period of their blooming ensure a continuous, unbroken feeding band for Apoidea from early spring till late summer.

3. Boundary strips, fallows and bush communities form in agricultural landscape refuge areas for bee plants as well as for valuable, rare elements of flora, hence they positively influence the biodiversity.

4. The dynamic succession observed on fallows caused a decrease in bee flora in the successive years of study. The treatments including sowing fallows with nectariferous and polleniferous species would enrich generally weak flows in highly agricultural landscape.

Acknowledgements. The authors would like to thank the Department of Geomorphology, Institute of Earth Science, Maria Curie-Skłodowska University in Lublin, for making the air photo available.

\section{REFERENCE}

Corbet S. A., Williams I. H., Osborne J. L., 1991. Bees and the pollination of crop and wild flowers in the European Communities. Bee World, 72(2): 4759.

Dostatny D., 2006. Traditional farming as a tool for weed con servation. 1 st European Congress of Conservation Biol ogy. 2226 August Eger Hungary:23 24 .

Jabłoński B., 2000. O potrzebie i możliwościach poprawy pożytków pszczelich. / On the need and possibilities of improvement of pollen flows. Oddział Pszczelnictwa ISK, Puławy.

Jabłoński B., Kołtowski Z., 1995. An attempt to outli ne the changes in the forage base of bees in Poland. In: Changes in fauna of wild bees in Europe. Pedagogical Univ., Bydgoszcz.

Matuszkiewicz 2001. Przewodnik do oznaczania zbiorowisk roślinnych Polski. /Guide for indentification of plant communities in Poland. Wyd. Nauk. PWN. Warszawa.

Mirek Z., Piekoś Mirkowa H., Zając A., Zając M., 2002. Flowering plants and pteridophytes of Poland. A checlist. Biodiversity of Poland 1: 1-442. In. Szafer In stitute of Botany, Polish Academy of Sciences, Kraków.

Parrish J. A. D., Bazzaz F. A., 1979. Difference in pol lination niche relationships in early and late succesional plant communities. Ecology, 60(3) 597610.

Turski R., Uziak S., Zawadzki S., 1993. Gleby. Śro dowisko przyrodnicze Lubelszczyzny. Soils. The natural environment of the Lublin region. Lub. Tow. Naukowe, Lublin.

Warakomska Z., 1995. The composition of polleniferous plants of the Kraków region in the microscopic ananysis of pollen loads of the honeybee. In Changes in fauna of wild bees in Europe. Pedagogical Univ., Bydgoszcz.

Warakomska Z., 1997. Pollen spectrum of multifloral honey of Lublin region. LTN: 170177.

Wróblew sk a A., 2002. Rośliny pożytkowe Podlasia w świetle analizy pyłkowej produktów pszczelich. / Flow plants of the Podlasie region in the light of pollen analysis of honey products. Rozprawy naukowe AR Lublin.

Van Opstal A. J., 2000. The architecture of the Pan European Ecological Network. National Reference Centre for Natu re Management, Ministry of Agriculture, Wageningen.

Zając A., 1979. Pochodzenie archeofitów występujących w Polsce. / The origin of archeophytes occurring in Po land. Rozp. Hab. Univ. Jagiel. 29: 1213.

Zając A., Tokarska Guzik B., 1998. Kenophytes in the flora of Poland: list, status and origin. W: Synantropiza tion of plant cover in new Polish research. Phytocoenosis 10 (N. S.) Suppl. Cartogr. Geobot. 9: 107116 


\section{Miedze i zadrzewienia śródpolne jako refugia roślin pożytkowych $w$ krajobrazie rolniczym}

\section{Streszczenie}

Badania prowadzono w latach 2002-2006 na terenie gmin Jastków i Konopica położonych w bezpośrednim sąsiedztwie miasta Lublina. Dokonano inwentaryzacji roślinności w obrębie miedz, gruntów odłogowanych oraz zadrzewień śródpolnych ze szczególnym uwzględnieniem roślinności dostarczającej pożytku nektarowego i pyłkowego. Ogółem w obrębie obserwowanych biotopów zanotowano 214 gatunków roślin, w tym $80 \%$ stanowiły taksony pożytkowe. W analizowanej florze przeważały apofity - 78\% nad gatunkami obcymi (kenofitami). We florze miedz i zadrzewień śródpolnych przewagę stanowiły gatunki wieloletnie. Taksony krótkotrwałe $(40 \%$ - jednoroczne i $15 \%$ dwuletnie) dominowały na gruntach odłogowanych. Zdecydowana większość gatunków pożytkowych reprezentuje zbiorowiska łąkowe, segetalne i ruderalne. Większość z nich występuje w luźnych skupiskach, ale ich sukcesywne kwitnienie zapewnia, na badanym terenie, ciagłość taśmy pokarmowej od wczesnej wiosny do późnego lata. Miedze, zadrzewienia śródpolne oraz odłogi, stanowią ważne w krajobrazie rolniczym ostoje roślinności pożytkowej jednocześnie zwiększają walor bioróżnorodności florystycznej tych terenów. Wydaje się, że ten typ siedlisk można wykorzystywać do podsiewania gatunków nektarodajnych i pyłkodajnych w celu wzbogacania generalnie słabych pożytków na terenach rolniczych. 
